\title{
Synthesis and antitumor activity of some novel thiophene, pyrimidine, coumarin, pyrazole and pyridine derivatives
}

\author{
MOHAMMED ALBRATTY ${ }^{1}$ \\ KARAM AHMED EL-SHARKAW $Y^{1,2 *}$ \\ SHAMSHER ALAM ${ }^{1}$ \\ ${ }^{1}$ Department of Pharmaceutical \\ Chemistry, College of Pharmacy \\ Jazan University, P.O. Box 114 \\ Jazan 45142, Saudi Arabia \\ ${ }^{2}$ Department of Organic Chemistry \\ Faculty of Biotechnology \\ October University for Modern \\ Sciences and Arts (MSA) \\ El-Wahat Road \\ 6 October City, Egypt
}

2-Cyano-N-(thiazol-2-yl) acetamide (2a) and 2-cyano- $N$-(oxazol2-yl) acetamide (2b) were obtained via the reaction of ethyl cyanoacetate with either 2-aminothiazole (1a) or 2-aminooxazole (1b). The formed products were directed toward the reaction with cyclopentanone and elemental sulfur in the presence of triethylamine to give cyclopenta[ $b]$ thiophene derivatives $(\mathbf{3} \mathbf{a}, \mathbf{b})$. The latter products were reacted with either ethyl cyanoacetate or malononitrile to form compounds $\mathbf{4} \mathbf{a}, \mathbf{b}$ and $\mathbf{5} \mathbf{a}, \mathbf{b}$, respectively. Compounds $\mathbf{4} \mathbf{a}, \mathbf{b}$ were aimed at synthesizing some heterocyclic compounds; thus internal cyclization reactions were introduced to form compounds $\mathbf{6} \mathbf{a}, \mathbf{b}$. Also, compounds $\mathbf{4} \mathbf{a}, \mathbf{b}$ reacted with salicylaldehyde, hydrazine derivatives and either urea or thiourea to produce coumarin derivatives $(7 \mathbf{a}, \mathbf{b})$, pyrazole derivatives (8a-d) and pyrimidine derivatives (9a-d), respectively. Reaction of either benzaldehyde or benzene diazonium chloride (11) with compounds $\mathbf{4} \mathbf{a}, \mathbf{b}$ afforded compounds $\mathbf{1 0} \mathbf{a}, \mathbf{b}$ and $\mathbf{1 2} \mathbf{a}, \mathbf{b}$, respectively. On the other hand, compounds $\mathbf{5} \mathbf{a}, \mathbf{b}$ underwent internal cyclization to form pyrimidine derivatives $\mathbf{1 3} \mathbf{a}, \mathbf{b}$. Also, when compounds $\mathbf{5 a}, \mathbf{b}$ reacted with either ethyl cyanoacetate or malononitrile, they gave pyridine derivatives (15a-d) through the formation of intermediates (14a-d). Finally, formation of fused pyrimidine derivatives $(\mathbf{1 7} \mathbf{a}, \mathbf{b})$ was achieved through the reaction of compounds $\mathbf{5} \mathbf{a}, \mathbf{b}$ and salicylaldehyde applying two different pathways. The first pathway used a catalytic amount of piperidine to form compounds $\mathbf{1 6} \mathbf{a}, \mathbf{b}$; the latter products underwent cyclization to give compounds $\mathbf{1 7 a}, \mathbf{b}$. The second pathway, using a catalytic amount of sodium ethoxide solution directly in one step, afforded compounds $\mathbf{1 7}$ a,b. Structures of the newly synthesized compounds were established using IR, ${ }^{1} \mathrm{H}$ NMR, ${ }^{13} \mathrm{C}$ NMR and mass spectrometry and their antitumor activity was investigated. Some of these compounds showed promising inhibitory effects on three different cell lines. However, fused pyrimidine acetonitrile derivatives 6a and $\mathbf{6 b}$ exerted the highest inhibitory effect, comparable to that of doxorubicin.

Keywords: thiophene, pyrimidine, coumarin, pyrazole, pyridine, antitumor activity 
Amino-thiophene derivatives were shown to be one of the most important groups of heterocyclic compounds with a wide spectrum of biological activities such as antitumor (1), antimitotic (2) and antiviral (3), in addition to either thiazol-amide with fungicidal activity (4) or oxazole-amide with antimicrobial activity (5). Furthermore, thieno[2,3- $d]$ pyrimidine derivatives show antibacterial (6) and antiproliferative activity (7). Also, chromene-3-carboxamide acts as an anti-Helicobacter pylori agent (8) and pyrazole derivatives have a specific effect with favorable antitumor activity (9). In addition, pyridine derivatives show anticonvulsant and anti-inflammatory (10), potential antitubercular (11) and anticonvulsant activity (12), among others.

In this article, we have described the synthesis of new heterocyclic compounds, thiophene, pyrimidine, coumarin, pyrazole and pyridine derivatives, in an attempt to improve the antitumor activity against three different cell lines: MCF-7 (breast adenocarcinoma), NCI-H460 (non-small cell lung cancer), and SF-268 (CNS cancer).

\section{EXPERIMENTAL}

Melting points were determined in open capillaries and are not corrected. A Yanaco CHN CORDER MT-6 elemental analyzer (Japan) was used. IR spectra were recorded in $\mathrm{KBr}$ pellets using a PA-9721 IR spectrophotometer (Shimadzu, Japan). A Jeol $300 \mathrm{MHz}$ (Japan) instrument was used to record the ${ }^{1} \mathrm{H}$ NMR and ${ }^{13} \mathrm{C}$ NMR spectra. $\mathrm{CD}_{3} \mathrm{SOCD}_{3}$ was used as a solvent and TMS as internal standard. Chemical shifts were expressed as $\delta$ (ppm). Kratos (75 eV) MS equipment (Germany) was used for mass spectra recording.

The synthetic pathways used are represented in Schemes 1-4 while the physicochemical and spectral data of the newly synthesized compounds are given in Tables I and II.

\section{Syntheses}

2-Cyano-N-(thiazol-2-yl)acetamide (2a) and 2-cyano-N-(oxazol-2-yl)acetamide (2b). - To a solution of either 2-aminothiazole (1a) $(5.007 \mathrm{~g}, 0.05 \mathrm{~mol})$ or 2-aminooxazole (1b) $(4.204 \mathrm{~g}$, $0.05 \mathrm{~mol})$ in $50 \mathrm{~mL}$ of absolute ethanol containing triethylamine $(0.5 \mathrm{~mL})$ as a catalyst,

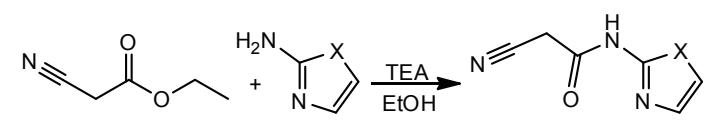

$1 \mathrm{a}, \mathrm{b}$
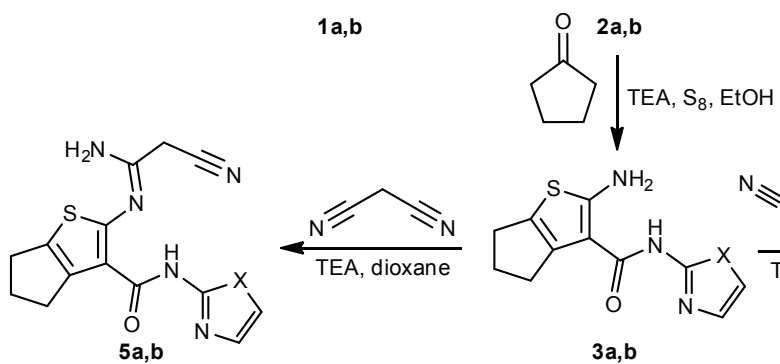

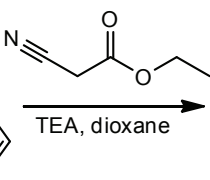

Scheme 1

$$
\text { a } X=S
$$<smiles>[X]c1cc[nH]c1NC(=O)c1c(NC(=O)CC#N)sc2c1CCC2</smiles> 
ethyl cyanoacetate $(5.656 \mathrm{~g}, 0.05 \mathrm{~mol})$ was added and the reaction mixture was heated under reflux for $3 \mathrm{~h}$, cooled and poured onto ice. Conc. $\mathrm{HCl}$ (a few drops) was added. The formed precipitate was filtered out and recrystallized from 1,4 dioxane to afford the desired product.

2-Amino-N-(thiazol-2-yl)-5,6-dihydro-4H-cyclopenta[b]thiophene-3-carboxamide (3a) and 2-amino-N-(oxazol-2-yl)-5,6-dihydro-4H-cyclopenta[b]thiophene-3-carboxamide (3b). - To a solution of either compound $\mathbf{2 a}(5.016 \mathrm{~g}, 0.03 \mathrm{~mol})$ or $\mathbf{2 b}(4.534 \mathrm{~g}, 0.03 \mathrm{~mol})$ in $50 \mathrm{~mL}$ of absolute EtOH containing triethylamine $(1.0 \mathrm{~mL})$, cyclopentanone $(2.524 \mathrm{~g}, 0.03 \mathrm{~mol})$ and elemental sulfur $(0.96 \mathrm{~g}, 0.03 \mathrm{~mol})$ were added. The whole reaction mixture was heated under reflux for $2 \mathrm{~h}$, then poured into an ice/water mixture containing a few drops of concentrated hydrochloric acid and the formed solid product, in each case, was collected by filtration and recrystallized from absolute ethanol.

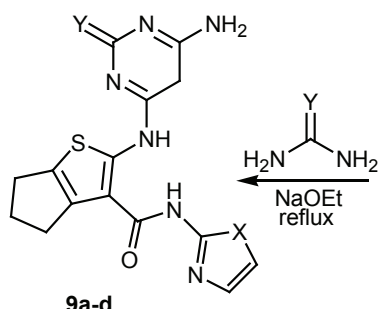

9a-d

a $X=S, Y=O$

b $X=O, Y=O$

c $\mathrm{X}=\mathrm{S}, \mathrm{Y}=\mathrm{S}$

d $X=O, Y=S$
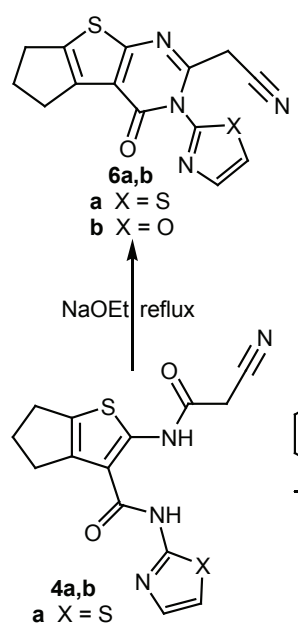

$$
\text { b } X=O
$$$$
\mathrm{RNHNH}_{2} \underset{\begin{array}{c}
\text { piperidine } \\
\text { etanol }
\end{array}}{\text { etanol }}
$$
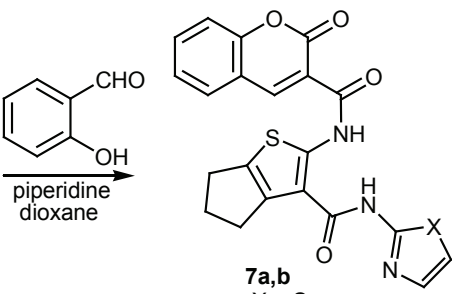

a $X=S$

b $X=0$<smiles>[R]n1ccc(Nc2sc3c(c2C(=O)Nc2cc(N)n([R])n2)CCC3)n1</smiles>

Scheme 2 


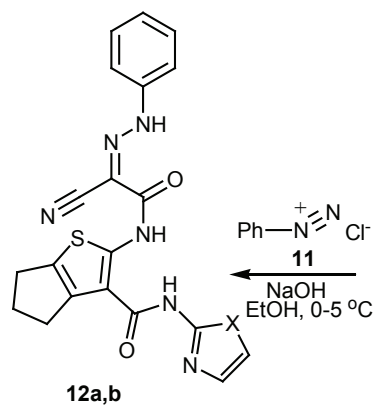<smiles>[X]c1cc[nH]c1NC(=O)c1c(NC(=O)CC#N)sc2c1CCC2</smiles><smiles>O=Cc1ccccc1</smiles><smiles></smiles><smiles>[R3][Y4](=O)O</smiles>

Scheme 3

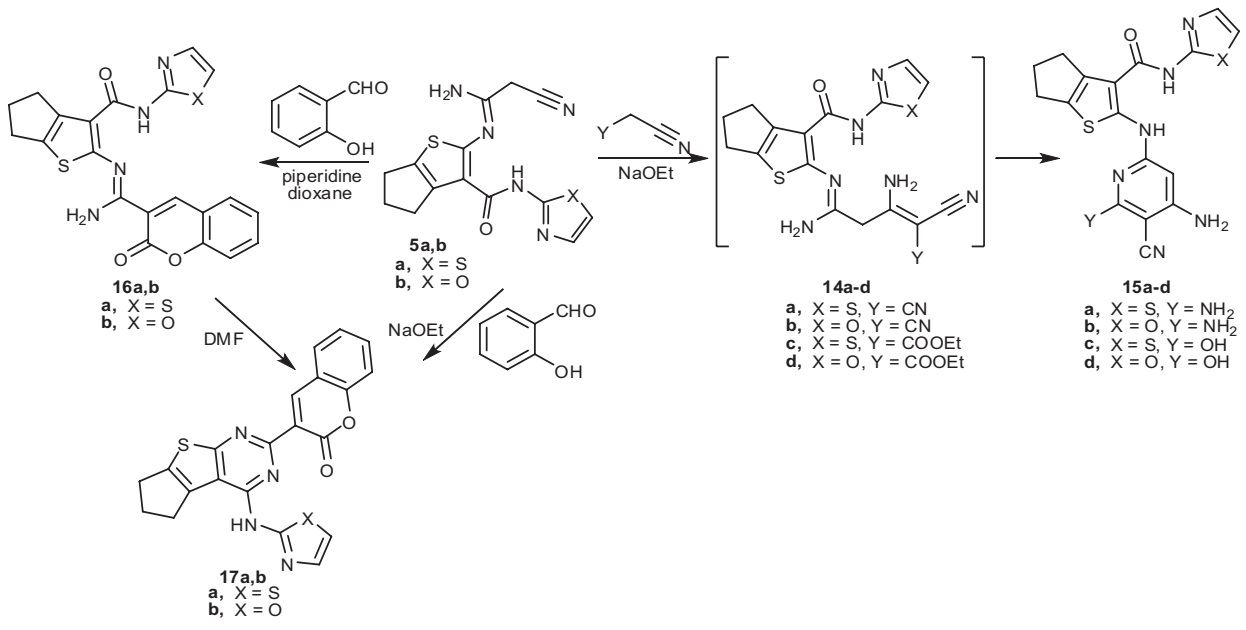

Scheme 4

2-(2-Cyanoacetamido)-N-(thiazol-2-yl)-5,6-dihydro-4H-cyclopenta[b] thiophene-3-carboxamide $(\mathbf{4 a})$ and 2-(2-cyanoacetamido)-N-(oxazol-2-yl)-5,6-dihydro-4H-cyclopenta[b]thiophene-3carboxamide (4b). - To a solution of either compound $3 \mathbf{a}(5.307 \mathrm{~g}, 0.02 \mathrm{~mol})$ or $3 \mathbf{b}(4.986 \mathrm{~g}, 0.02$ $\mathrm{mol})$ in 1,4-dioxane $(50 \mathrm{~mL})$ containing triethylamine $(0.5 \mathrm{~mL})$, ethyl cyanoacetate $(2.262 \mathrm{~g}$, $0.02 \mathrm{~mol}$ ) was added. The resulting reaction mixture was heated under reflux for $3 \mathrm{~h}$, cooled and poured into an ice/water mixture with a few drops of conc. $\mathrm{HCl}$ added. The precipitate that formed was filtered and recrystallized from absolute ethanol. 
Table I. Physicochemical data for synthesized compounds

\begin{tabular}{|c|c|c|c|c|c|c|c|}
\hline \multirow[t]{2}{*}{ Compd. } & \multirow{2}{*}{$\begin{array}{l}\text { Mol. formula } \\
\qquad\left(M_{\mathrm{r}}\right)\end{array}$} & \multirow{2}{*}{$\begin{array}{l}\text { M. p. } \\
\left({ }^{\circ} \mathrm{C}\right)\end{array}$} & \multirow{2}{*}{$\begin{array}{c}\text { Yield } \\
(\%)\end{array}$} & \multicolumn{4}{|c|}{ Calcd./found (\%) } \\
\hline & & & & C & $\mathrm{H}$ & $\mathrm{N}$ & S \\
\hline \multirow{2}{*}{$2 a$} & $\mathrm{C}_{6} \mathrm{H}_{5} \mathrm{~N}_{3} \mathrm{OS}$ & \multirow{2}{*}{$229-231$} & \multirow{2}{*}{74} & 43.10 & 3.01 & 25.13 & 19.18 \\
\hline & 167.19 & & & 43.32 & 3.23 & 24.98 & 18.96 \\
\hline \multirow{2}{*}{$2 b$} & $\mathrm{C}_{6} \mathrm{H}_{5} \mathrm{~N}_{3} \mathrm{O}_{2}$ & \multirow{2}{*}{ 201-202 } & \multirow{2}{*}{70.5} & 47.69 & 3.33 & 27.81 & \multirow{2}{*}{-} \\
\hline & 151.12 & & & 47.85 & 3.11 & 27.56 & \\
\hline \multirow{2}{*}{$3 a$} & $\mathrm{C}_{11} \mathrm{H}_{11} \mathrm{~N}_{3} \mathrm{OS}_{2}$ & \multirow{2}{*}{$169-171$} & \multirow{2}{*}{81} & 49.79 & 4.18 & 15.84 & 24.17 \\
\hline & 265.35 & & & 49.55 & 4.02 & 15.99 & 24.39 \\
\hline \multirow{2}{*}{$3 b$} & $\mathrm{C}_{11} \mathrm{H}_{11} \mathrm{~N}_{3} \mathrm{O}_{2} \mathrm{~S}$ & \multirow{2}{*}{$209-211$} & \multirow{2}{*}{77} & 53.00 & 4.45 & 16.86 & 12.86 \\
\hline & 249.29 & & & 53.25 & 4.22 & 16.57 & 12.70 \\
\hline \multirow{2}{*}{$4 a$} & $\mathrm{C}_{14} \mathrm{H}_{12} \mathrm{~N}_{4} \mathrm{O}_{2} \mathrm{~S}_{2}$ & \multirow{2}{*}{ 242-244 } & \multirow{2}{*}{66} & 50.59 & 3.64 & 16.86 & 19.29 \\
\hline & 332.4 & & & 50.40 & 3.44 & 16.63 & 19.13 \\
\hline \multirow{2}{*}{$4 b$} & $\mathrm{C}_{14} \mathrm{H}_{12} \mathrm{~N}_{4} \mathrm{O}_{3} \mathrm{~S}$ & \multirow{2}{*}{$186-188$} & \multirow{2}{*}{71} & 53.16 & 3.82 & 17.71 & 10.14 \\
\hline & 316.34 & & & 53.41 & 3.59 & 17.50 & 9.88 \\
\hline \multirow{2}{*}{$5 a$} & $\mathrm{C}_{14} \mathrm{H}_{13} \mathrm{~N}_{5} \mathrm{OS}_{2}$ & \multirow{2}{*}{ 195-196 } & \multirow{2}{*}{73} & 50.74 & 3.95 & 21.13 & 19.35 \\
\hline & 331.42 & & & 50.96 & 3.66 & 21.35 & 19.17 \\
\hline \multirow{2}{*}{$5 b$} & $\mathrm{C}_{14} \mathrm{H}_{13} \mathrm{~N}_{5} \mathrm{O}_{2} \mathrm{~S}$ & 158-160 & 61 & 53.32 & 4.16 & 22.21 & 10.17 \\
\hline & 315.35 & $100-100$ & 01 & 53.15 & 3.97 & 22.49 & 10.47 \\
\hline $6 a$ & $\mathrm{C}_{14} \mathrm{H}_{10} \mathrm{~N}_{4} \mathrm{OS}_{2}$ & $211-213$ & 76 & 53.49 & 3.21 & 17.82 & 20.40 \\
\hline $0 \mathrm{a}$ & 314.39 & $211-210$ & & 53.77 & 3.45 & 17.55 & 20.16 \\
\hline $6 \mathrm{~b}$ & $\mathrm{C}_{14} \mathrm{H}_{10} \mathrm{~N}_{4} \mathrm{O}_{2} \mathrm{~S}$ & $227-229$ & 71 & 56.37 & 3.38 & 18.78 & 10.75 \\
\hline 000 & 298.32 & $228=223$ & 11 & 56.58 & 3.52 & 18.99 & 10.46 \\
\hline $7 a$ & $\mathrm{C}_{21} \mathrm{H}_{15} \mathrm{~N}_{3} \mathrm{O}_{4} \mathrm{~S}_{2}$ & 171-173 & 65 & 57.65 & 3.46 & 9.60 & 14.66 \\
\hline & 437.49 & & & 57.95 & 3.19 & 9.83 & 14.91 \\
\hline $7 \mathrm{~b}$ & $\mathrm{C}_{21} \mathrm{H}_{15} \mathrm{~N}_{3} \mathrm{O}_{5} \mathrm{~S}$ & $150-151$ & 61 & 59.85 & 3.59 & 9.97 & 7.61 \\
\hline & 421.43 & 160101 & 01 & 60.12 & 3.35 & 9.73 & 7.91 \\
\hline $8 a$ & $\mathrm{C}_{14} \mathrm{H}_{14} \mathrm{~N}_{6} \mathrm{OS}_{2}$ & $123-125$ & 62 & 48.54 & 4.07 & 24.26 & 18.51 \\
\hline oa & 346.43 & $120-123$ & & 48.75 & 3.85 & 24.02 & 18.23 \\
\hline $8 \mathrm{~b}$ & $\mathrm{C}_{14} \mathrm{H}_{14} \mathrm{~N}_{6} \mathrm{O}_{2} \mathrm{~S}$ & 155-157 & 58 & 50.90 & 4.27 & 25.44 & 9.71 \\
\hline 0 & 330.36 & & 50 & 51.13 & 4.49 & 25.67 & 9.45 \\
\hline $8 c$ & $\mathrm{C}_{20} \mathrm{H}_{18} \mathrm{~N}_{6} \mathrm{OS}_{2}$ & 178-180 & 55 & 56.58 & 4.29 & 19.89 & 15.18 \\
\hline or & 422.53 & $170-100$ & 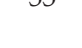 & 56.29 & 4.04 & 19.65 & 15.34 \\
\hline $8 d$ & $\mathrm{C}_{20} \mathrm{H}_{18} \mathrm{~N}_{6} \mathrm{O}_{2} \mathrm{~S}$ & $218-220$ & 51 & 59.10 & 4.46 & 20.68 & 7.89 \\
\hline (14 & 406.46 & 210220 & $J_{1}$ & 59.38 & 4.21 & 20.95 & 7.61 \\
\hline
\end{tabular}




\begin{tabular}{|c|c|c|c|c|c|c|c|}
\hline \multirow{2}{*}{$9 a$} & & \multirow{2}{*}{ 190-191 } & \multirow{2}{*}{52} & 48.11 & 3.77 & 22.44 & 17.13 \\
\hline & 374.44 & & & 48.36 & 3.53 & 22.33 & 17.36 \\
\hline \multirow{2}{*}{$9 b$} & $\mathrm{C}_{15} \mathrm{H}_{14} \mathrm{~N}_{6} \mathrm{O}_{3} \mathrm{~S}$ & \multirow{2}{*}{ 163-165 } & \multirow{2}{*}{48} & 50.27 & 3.94 & 23.45 & 8.95 \\
\hline & 358.38 & & & 50.54 & 3.68 & 23.16 & 8.66 \\
\hline \multirow{2}{*}{$9 c$} & $\mathrm{C}_{15} \mathrm{H}_{14} \mathrm{~N}_{6} \mathrm{OS}_{3}$ & \multirow{2}{*}{ 140-142 } & \multirow{2}{*}{57} & 46.14 & 3.61 & 21.52 & 24.63 \\
\hline & 390.51 & & & 46.44 & 3.90 & 21.23 & 24.40 \\
\hline \multirow{2}{*}{$9 d$} & $\mathrm{C}_{15} \mathrm{H}_{14} \mathrm{~N}_{6} \mathrm{O}_{2} \mathrm{~S}_{2}$ & \multirow{2}{*}{$221-223$} & \multirow{2}{*}{55} & 48.11 & 3.77 & 22.44 & 17.13 \\
\hline & 374.44 & & & 48.34 & 3.99 & 22.16 & 17.42 \\
\hline \multirow{2}{*}{$10 a$} & $\mathrm{C}_{21} \mathrm{H}_{16} \mathrm{~N}_{4} \mathrm{O}_{2} \mathrm{~S}_{2}$ & \multirow{2}{*}{$148-150$} & \multirow{2}{*}{59} & 59.98 & 3.84 & 13.32 & 15.25 \\
\hline & 420.51 & & & 60.24 & 3.56 & 13.12 & 15.42 \\
\hline \multirow{2}{*}{$10 \mathrm{~b}$} & $\mathrm{C}_{21} \mathrm{H}_{16} \mathrm{~N}_{4} \mathrm{O}_{3} \mathrm{~S}$ & \multirow{2}{*}{$112-114$} & \multirow{2}{*}{53} & 62.36 & 3.99 & 13.85 & 7.93 \\
\hline & 404.44 & & & 62.18 & 3.68 & 13.61 & 8.15 \\
\hline \multirow{2}{*}{$12 a$} & $\mathrm{C}_{20} \mathrm{H}_{16} \mathrm{~N}_{6} \mathrm{O}_{2} \mathrm{~S}_{2}$ & \multirow{2}{*}{ 131-133 } & \multirow{2}{*}{64} & 55.03 & 3.69 & 19.25 & 14.69 \\
\hline & 436.51 & & & 55.29 & 3.39 & 19.02 & 14.41 \\
\hline \multirow{2}{*}{$12 b$} & $\mathrm{C}_{20} \mathrm{H}_{16} \mathrm{~N}_{6} \mathrm{O}_{3} \mathrm{~S}$ & \multirow{2}{*}{ 110-111 } & \multirow{2}{*}{55} & 57.13 & 3.84 & 19.99 & 7.63 \\
\hline & 420.44 & & & 57.41 & 3.99 & 19.71 & 7.42 \\
\hline \multirow{2}{*}{$13 a$} & $\mathrm{C}_{14} \mathrm{H}_{11} \mathrm{~N}_{5} \mathrm{~S}_{2}$ & \multirow{2}{*}{ 237-239 } & \multirow{2}{*}{66} & 53.65 & 3.54 & 22.35 & 20.46 \\
\hline & 313.40 & & & 53.35 & 3.32 & 22.62 & 20.19 \\
\hline $13 b$ & $\mathrm{C}_{14} \mathrm{H}_{11} \mathrm{~N}_{5} \mathrm{OS}$ & 217-219 & 60 & 56.55 & 3.73 & 23.55 & 10.78 \\
\hline & 297.34 & & & 56.83 & 3.96 & 23.31 & 10.53 \\
\hline $15 a$ & $\mathrm{C}_{17} \mathrm{H}_{15} \mathrm{~N}_{7} \mathrm{OS}_{2}$ & $215-217$ & 56 & 51.37 & 3.80 & 24.67 & 16.13 \\
\hline 100 & 397.48 & $210-213$ & - & 51.58 & 3.61 & 24.49 & 16.34 \\
\hline $15 b$ & $\mathrm{C}_{17} \mathrm{H}_{15} \mathrm{~N}_{7} \mathrm{O}_{2} \mathrm{~S}$ & $222-224$ & 51 & 53.53 & 3.96 & 25.71 & 8.41 \\
\hline & 381.41 & $22<-2<4$ & $J 1$ & 53.28 & 3.69 & 25.51 & 8.23 \\
\hline $15 c$ & $\mathrm{C}_{17} \mathrm{H}_{14} \mathrm{~N}_{6} \mathrm{O}_{2} \mathrm{~S}_{2}$ & $230-232$ & 53 & 51.24 & 3.54 & 21.09 & 16.09 \\
\hline & 398.46 & & & 51.03 & 3.28 & 20.87 & 16.32 \\
\hline $15 d$ & $\mathrm{C}_{17} \mathrm{H}_{14} \mathrm{~N}_{6} \mathrm{O}_{3} \mathrm{~S}$ & $300<$ & 48 & 53.40 & 3.69 & 21.98 & 8.39 \\
\hline & 382.40 & 300 & & 53.63 & 3.91 & 21.69 & 8.21 \\
\hline $16 a$ & $\mathrm{C}_{21} \mathrm{H}_{16} \mathrm{~N}_{4} \mathrm{O}_{3} \mathrm{~S}_{2}$ & 105-107 & 63 & 57.78 & 3.69 & 12.84 & 14.69 \\
\hline & 436.51 & & & 57.52 & 3.44 & 13.03 & 14.45 \\
\hline $16 \mathrm{~b}$ & $\mathrm{C}_{21} \mathrm{H}_{16} \mathrm{~N}_{4} \mathrm{O}_{4} \mathrm{~S}$ & 89-91 & 66 & 59.99 & 3.84 & 13.33 & 7.63 \\
\hline & 420.44 & 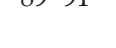 & 00 & 59.78 & 3.99 & 13.55 & 7.48 \\
\hline $17 a$ & $\mathrm{C}_{21} \mathrm{H}_{14} \mathrm{~N}_{4} \mathrm{O}_{2} \mathrm{~S}_{2}$ & 144-145 & 55 & 60.27 & 3.37 & 13.39 & 15.32 \\
\hline & 418.89 & & & 59.98 & 3.59 & 13.14 & 15.12 \\
\hline $17 b$ & $\mathrm{C}_{21} \mathrm{H}_{14} \mathrm{~N}_{4} \mathrm{O}_{3} \mathrm{~S}$ & 181-183 & 62 & 62.68 & 3.51 & 13.92 & 7.97 \\
\hline $1 / 0$ & 402.43 & $101-100$ & 02 & 62.95 & 3.28 & 13.74 & 7.67 \\
\hline
\end{tabular}




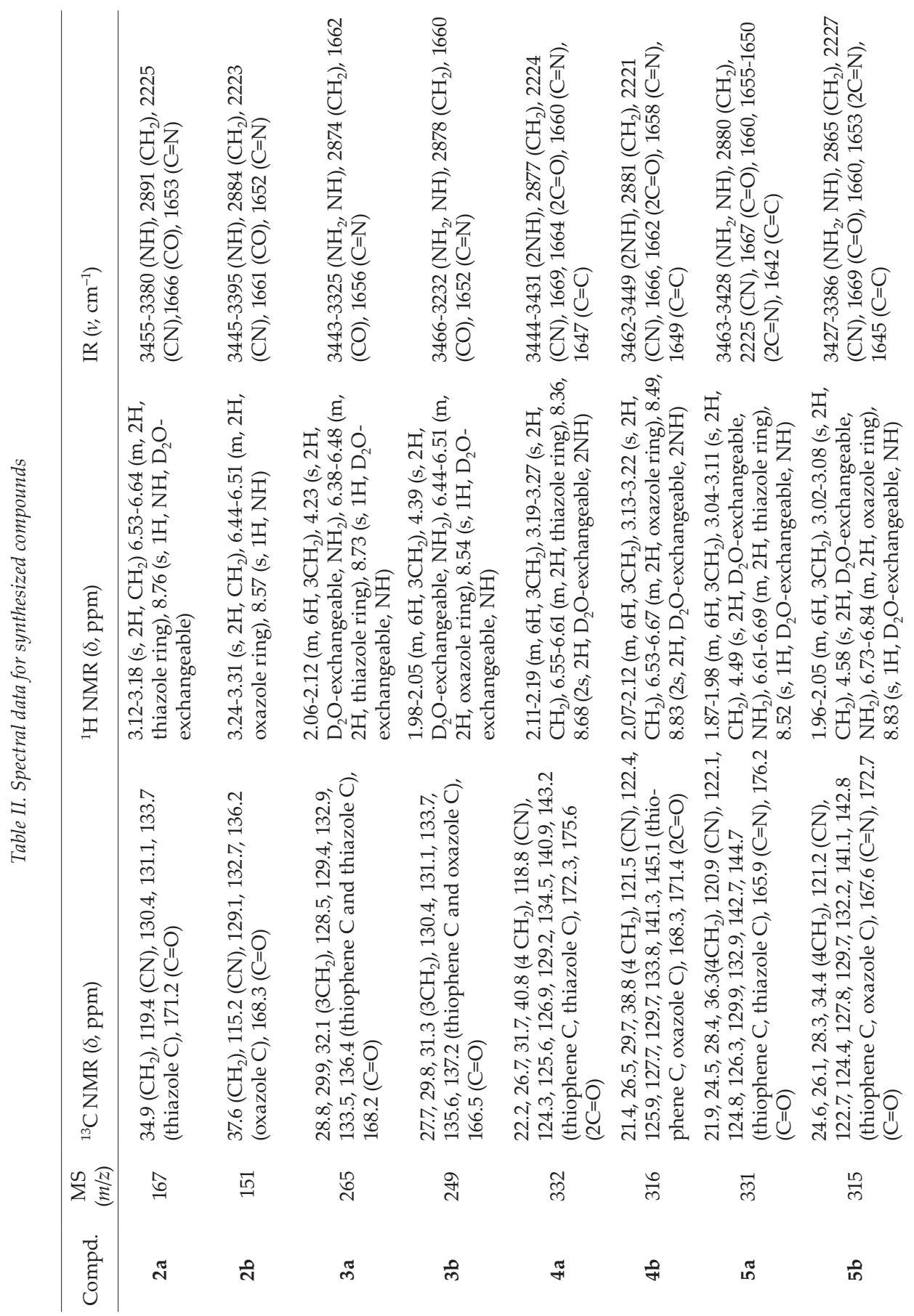




\begin{tabular}{|c|c|c|c|c|c|c|c|}
\hline $\begin{array}{l}\widehat{T} \\
\Xi \\
\hat{\Xi} \\
\cong\end{array}$ & 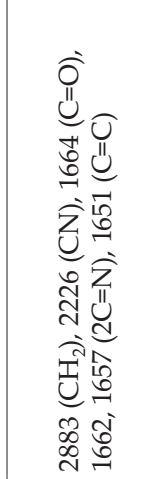 & 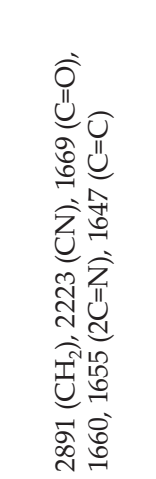 & 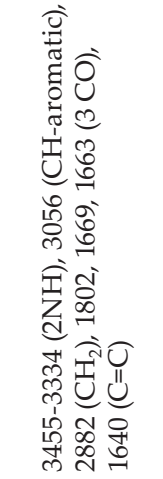 & 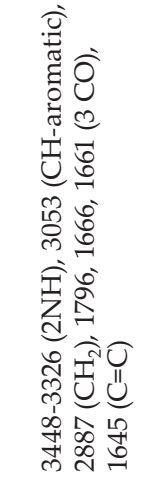 & 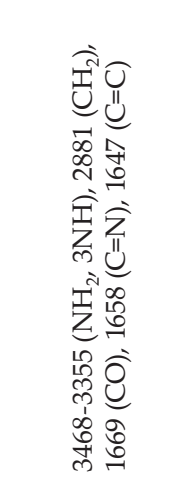 & 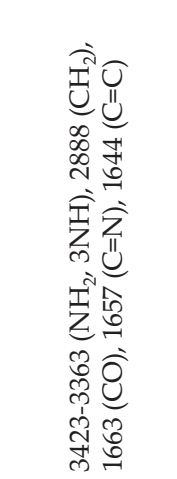 & 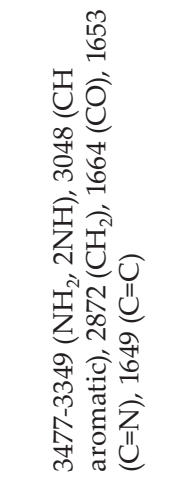 \\
\hline 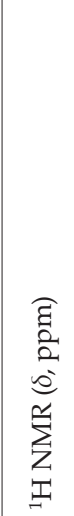 & 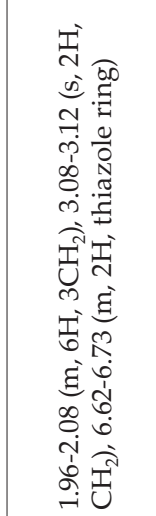 & 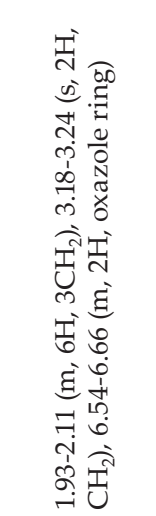 & 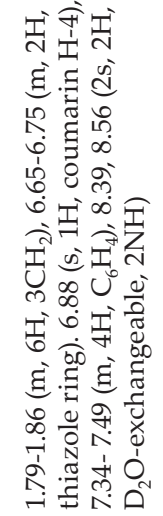 & 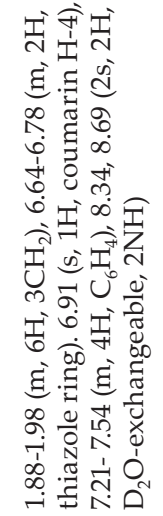 & 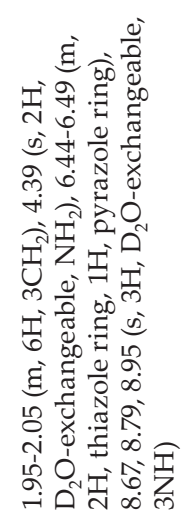 & 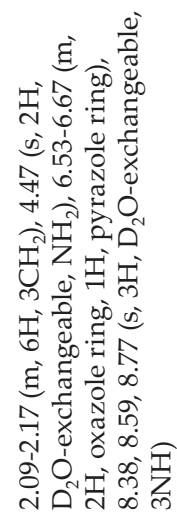 & 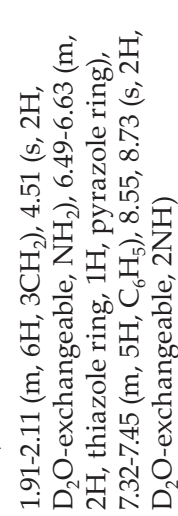 \\
\hline 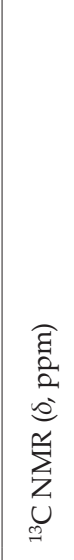 & 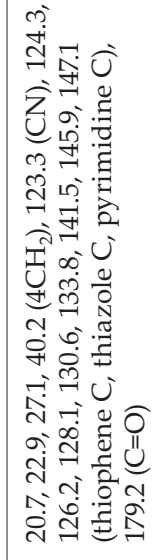 & 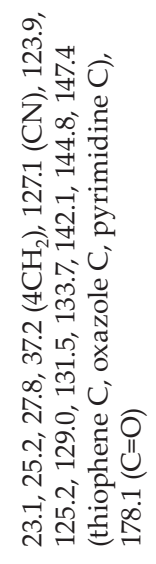 & 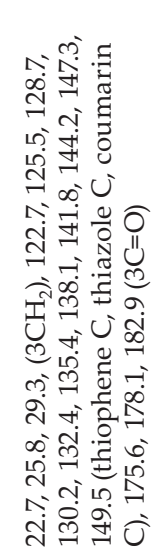 & 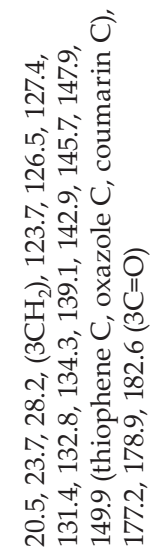 & 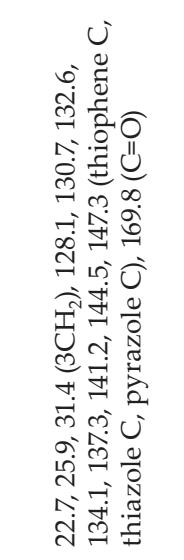 & 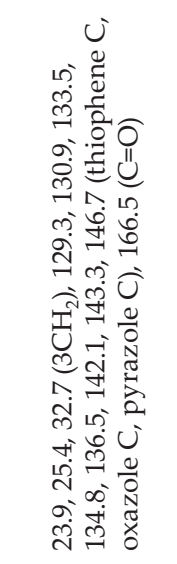 & 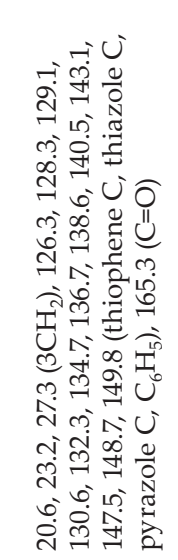 \\
\hline$\sum_{\Sigma}^{\infty} \frac{\mathbb{N}}{\Xi}$ & $\ddot{m}$ & $\stackrel{\infty}{\stackrel{N}{ }}$ & $\hat{q}$ & $\overline{7}$ & 胥 & $\bar{m}$ & สี \\
\hline & శే & 8 & ก็ & Rे & 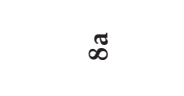 & D & $\infty$ \\
\hline
\end{tabular}




\begin{tabular}{|c|c|c|c|c|c|c|}
\hline 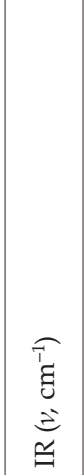 & 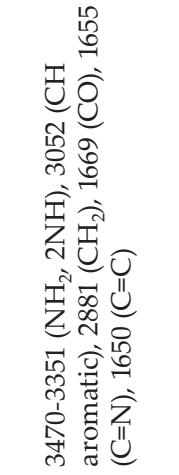 & 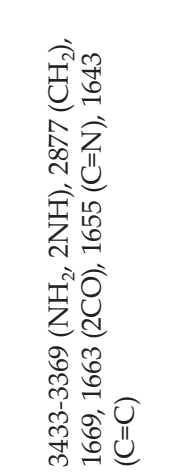 & 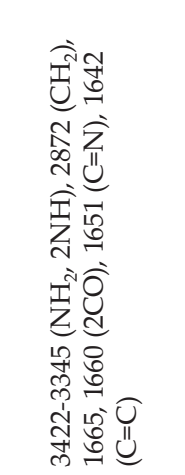 & 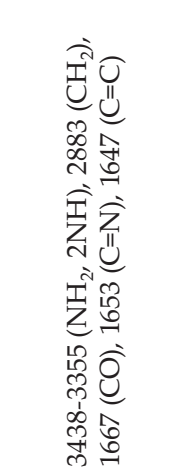 & 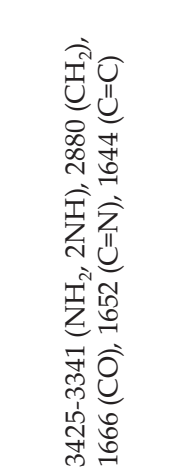 & 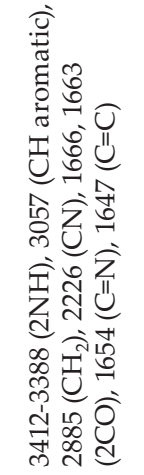 \\
\hline 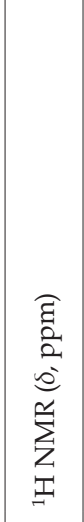 & 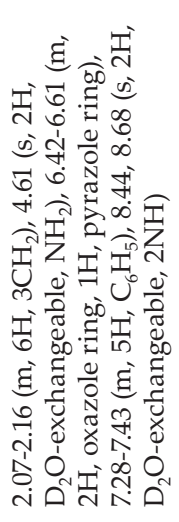 & 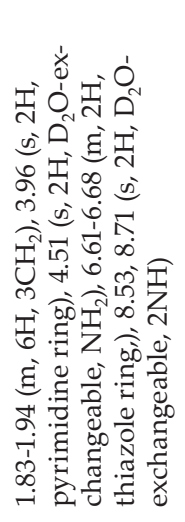 & 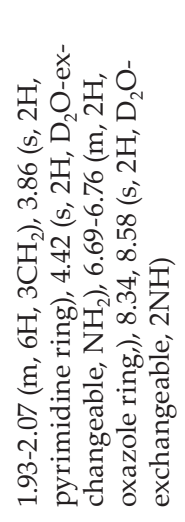 & 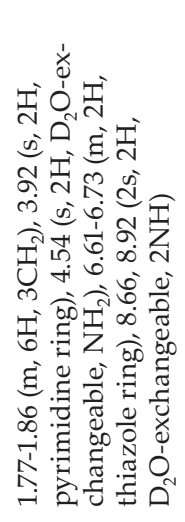 & 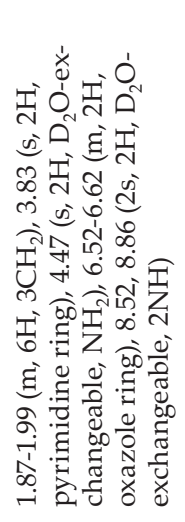 & 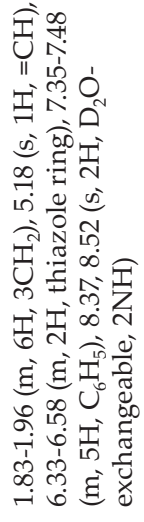 \\
\hline 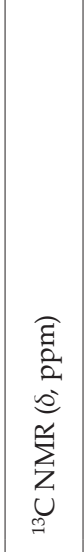 & 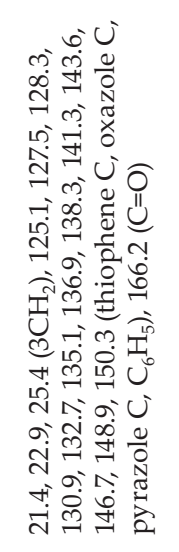 & 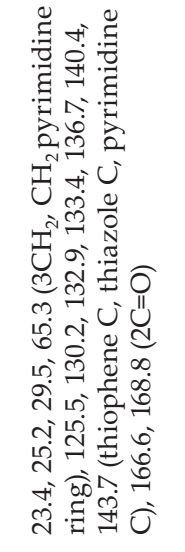 & 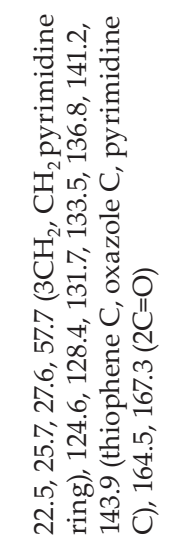 & 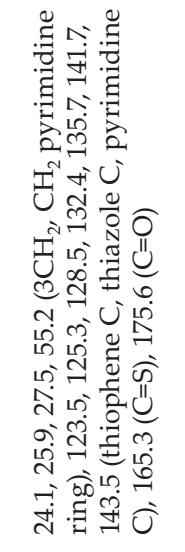 & 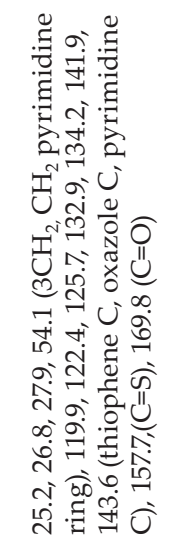 & 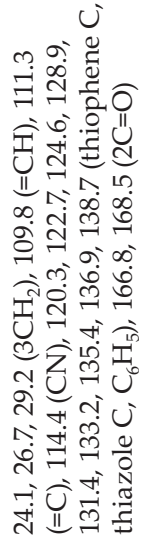 \\
\hline$\sum_{\Sigma}^{\infty} \frac{\pi}{\Sigma}$ & \& & 夰 & $\begin{array}{l}\infty \\
\stackrel{\infty}{\infty} \\
m\end{array}$ & ஓి & 劳 & ్ํ \\
\hline $\begin{array}{l}\text { Dे } \\
\text { ह् } \\
\end{array}$ & $\infty$ & శ็ & ने & o & నू & \\
\hline
\end{tabular}




\begin{tabular}{|c|c|c|c|c|c|c|c|}
\hline $\begin{array}{l}\widehat{T} \\
\text { ह } \\
\grave{\vec{\Xi}} \\
\cong\end{array}$ & 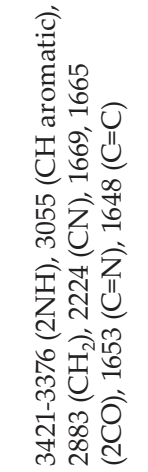 & 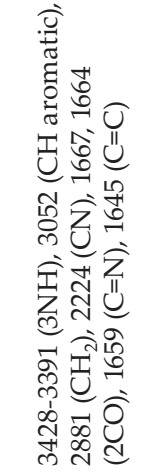 & 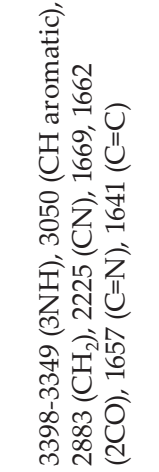 & 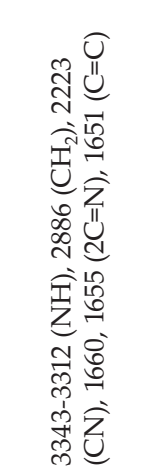 & 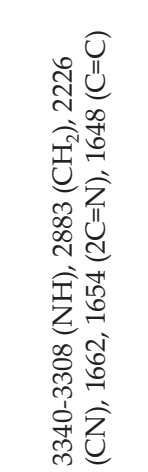 & 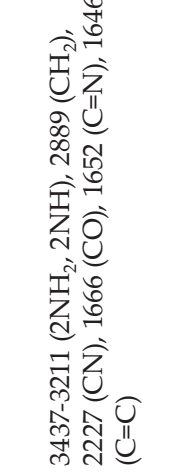 & 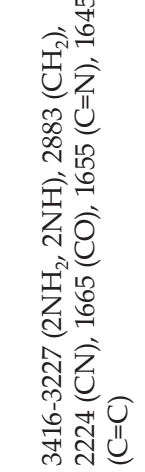 \\
\hline 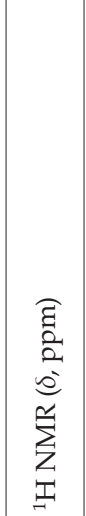 & 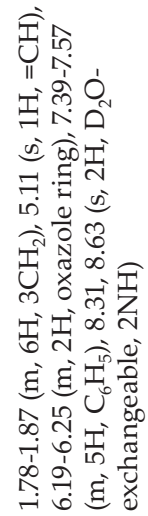 & 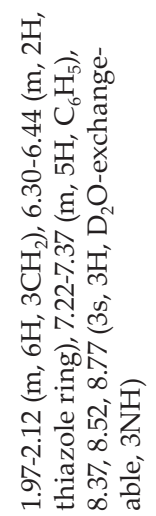 & 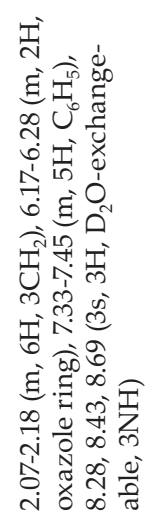 & 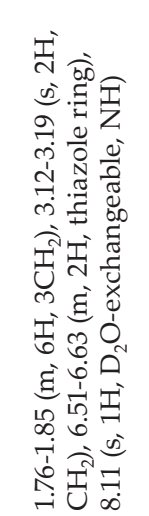 & 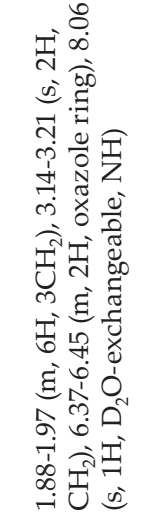 & 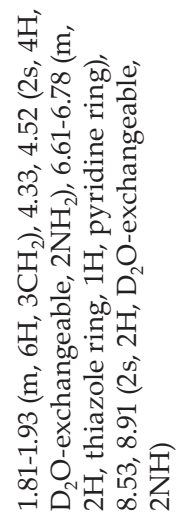 & 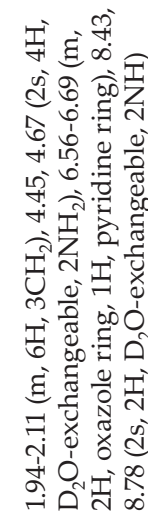 \\
\hline 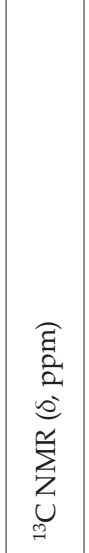 & 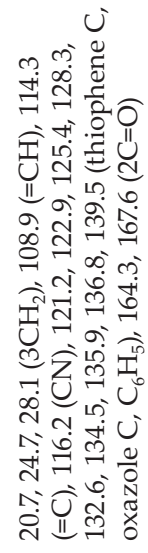 & 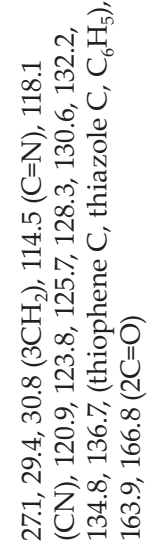 & 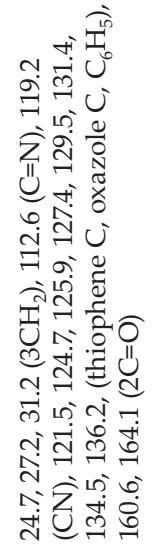 & 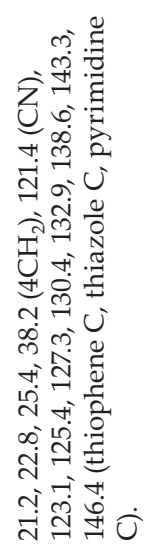 & 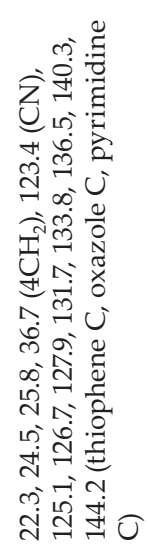 & 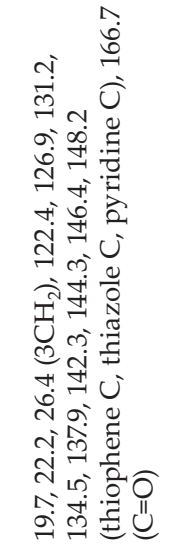 & 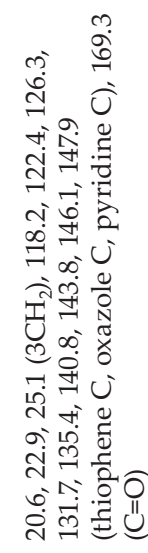 \\
\hline$\sum^{\infty} \frac{\pi}{3}$ & 亲 & ঙ্ণ & ఫิ & $\stackrel{m}{m}$ & ลे & ભે & $\vec{\infty}$ \\
\hline & $=$ & $\stackrel{\mathbb{N}}{2}$ & $\overrightarrow{\mathrm{N}}$ & 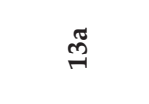 & $\bar{n}$ & 12 & 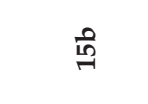 \\
\hline
\end{tabular}


2-(1-Amino-2-cyanoethylideneamino)-N-(thiazol-2-yl)-5,6-dihydro-4H-cyclopenta[b]thiophene-3-carboxamide (5a) and 2-(1-amino-2-cyanoethylideneamino)-N-(oxazol-2-yl)-5,6-dihydro$4 \mathrm{H}$-cyclopenta[b]thiophene-3-carboxamide $(5 \mathbf{b})$. - To a solution of either compound $3 \mathrm{a}(2.654$ $\mathrm{g}, 0.01 \mathrm{~mol})$ or $3 \mathrm{~b}(2.493 \mathrm{~g}, 0.01 \mathrm{~mol})$ in 1,4-dioxane $(40 \mathrm{~mL})$ containing triethylamine $(0.5$ $\mathrm{mL})$, malononitrile $(0.66 \mathrm{~g}, 0.01 \mathrm{~mol})$ was added. The reaction mixture was heated under reflux for $2 \mathrm{~h}$, cooled and poured into an ice/water mixture containing a few drops of conc. $\mathrm{HCl}$. The formed precipitate was filtered out and recrystallized from absolute ethanol.

2-(4-Oxo-3-(thiazol-2-yl)-3,4,6,7-tetrahydro-5H-cyclopenta[4,5]thieno[2,3-d]pyrimidin-2yl)acetonitrile (6a) and 2-(4-oxo-3-(oxazol-2-yl)-3,4,6,7-tetrahydro-5H-cyclopenta[4,5]thieno[2,3-d]pyrimidin-2-yl)acetonitrile (6b). - A suspension of either $4 \mathbf{a}(0.665 \mathrm{~g}, 0.002 \mathrm{~mol})$ or $4 \mathbf{b}$ $(0.633 \mathrm{~g}, 0.002 \mathrm{~mol})$ in sodium ethoxide $(0.002 \mathrm{~mol})$ [prepared by dissolving elemental sodium $(0.046 \mathrm{~g}, 0.002 \mathrm{~mol})$ in abs. EtOH $(40 \mathrm{~mL})]$ was heated over a boiling water bath for 6 $\mathrm{h}$ and then left to cool. The solid product formed upon pouring onto ice/water containing a few drops of hydrochloric acid $(10 \%)($ until $\mathrm{pH}=6)$. It was collected by filtration and then recrystallized from absolute ethanol.

$\mathrm{N}-(3-($ thiazol-2-yl-carbamoyl)-5,6-dihydro-4H-cyclopenta[b] thiophen-2-yl-2-oxo-2Hchromene-3-carboxamide (7a) and $\mathrm{N}$-(3-(oxazol-2-yl-carbamoyl)-5,6-dihydro-4H-cyclopenta[b] thiophen-2-yl)-2-oxo-2H-chromene-3-carboxamide ( $7 \boldsymbol{b})$. - To a solution of either compound $4 \mathbf{a}$ $(0.665 \mathrm{~g}, 0.002 \mathrm{~mol})$ or $4 \mathrm{~b}(0.633 \mathrm{~g}, 0.002 \mathrm{~mol})$ in 1,4-dioxane $(40 \mathrm{~mL})$ containing piperidine $(0.50 \mathrm{~mL})$, salicyladehyde $(0.244 \mathrm{~g}, 0.002 \mathrm{~mol})$ was added. The reaction mixture was heated under reflux for $6 \mathrm{~h}$ and then evaporated under vacuum. The remaining product was triturated with absolute ethanol and the formed solid product was collected by filtration and recrystallized from 1,4-dioxane.

2-(5-Amino-1H-pyrazol-3-ylamino)-N-(thiazol-2-yl)-5,6-dihydro-4H-cyclopenta[b] thiophene-3-carboxamide (8a), 2-(5-amino-1H-pyrazol-3-ylamino)-N-(oxazol-2-yl)-5,6-dihydro-4H-cyclopenta[b] thiophene-3-carboxamide (8b), 2-(5-amino-1-phenyl-1H-pyrazol-3-ylamino)-N-(thiazol-2-yl)-5,6-dihydro-4H-cyclopenta[b] thiophene-3-carboxamide $(8 \mathrm{c})$ and 2-(5-amino-1-phenyl1H-pyrazol-3-ylamino)-N-(oxazol-2-yl)-5,6-dihydro-4H-cyclopenta[b]thiophene-3-carboxamide $(8 d)$. - Either compound $4 \mathrm{a}(0.665 \mathrm{~g}, 0.002 \mathrm{~mol})$ or $4 \mathbf{b}(0.633 \mathrm{~g}, 0.002 \mathrm{~mol})$ was dissolved in absolute ethanol $(50 \mathrm{~mL})$ containing piperidine $(0.50 \mathrm{~mL})$, hydrazine hydrate $(0.1 \mathrm{~g}, 0.002$ mol) or phenylhydrazine $(0.216 \mathrm{~g}, 0.002 \mathrm{~mol})$ and the whole mixture was refluxed for $3 \mathrm{~h}$ and then poured into water containing ice and a few drops of concentrated $\mathrm{HCl}$. Suction filtration was then used to collect the coagulated precipitate; the formed solid product was recrystallized from ethanol.

2-(6-Amino-2-oxo-2,5-dihydropyrimidin-4-ylamino)-N-(thiazol-2-yl)-5,6-dihydro-4H-cyclo-

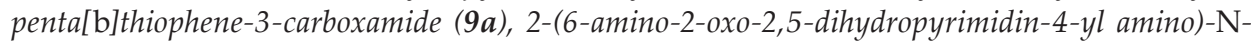
(oxazol-2-yl)-5,6-dihydro-4H-cyclopenta[b] thiophene-3-carboxamide (9b), 2-(6-amino-2-thioxo-2,5-dihydropyrimidin-4-ylamino)-N-(thiazol-2-yl)-5,6-dihydro-4H-cyclopenta[b]thiophene-3-carboxamide (9c) and 2-(6-amino-2-thioxo-2,5-dihydropyrimidin-4-ylamino)- $\mathrm{N}$-(oxazol-2-yl)-5,6-dihydro-4Hcyclopenta[b]thiophene-3-carboxamide $(9 d)$. - To a suspension of either compound $4 \mathrm{a}(0.665 \mathrm{~g}$, $0.002 \mathrm{~mol})$ or $4 \mathrm{~b}(0.633 \mathrm{~g}, 0.002 \mathrm{~mol})$ in sodium ethoxide $(0.002 \mathrm{~mol}, 50 \mathrm{~mL})$, either urea $(0.12 \mathrm{~g}$, $0.002 \mathrm{~mol})$ or thiourea $(0.152 \mathrm{~g}, 0.002 \mathrm{~mol})$ was added. The whole reaction mixture was heated over a boiling water bath for $3 \mathrm{~h}$, then poured onto ice/water containing a few drops 
of concentrated hydrochloric acid (until $\mathrm{pH}$ 6) and the solid product was collected by filtration and recrystallized from absolute ethanol.

2-(2-Cyano-3-phenylacrylamido)-N-(thiazol-2-yl)-5,6-dihydro-4H-cyclopenta[b] thiophene3-carboxamide (10a) and 2-(2-cyano-3-phenylacrylamido)-N-(oxazol-2-yl)-5,6-dihydro-4H-cyclopenta[b]thiophene-3-carboxamide (10b). - Either compound $4 \mathbf{a}(0.665 \mathrm{~g}, 0.002 \mathrm{~mol})$ or $\mathbf{4 b}$ (0.633 $\mathrm{g}, 0.002 \mathrm{~mol})$ was dissolved in $50 \mathrm{~mL}$ abs. EtOH with added piperidine $(0.50 \mathrm{~mL})$. Benzaldehyde $(0.21 \mathrm{~g}, 0.002 \mathrm{~mol})$ was added and the reaction mixture was heated under reflux for $3 \mathrm{~h}$ and then poured into ice-containing water with a few drops of conc. $\mathrm{HCl}$ added. The coagulated precipitate was collected by suction filtration and recrystallized from 1,4-dioxane.

2-(3-(Thiazol-2-yl-carbamoyl)-5,6-dihydro-4H-cyclopenta[b]thiophen-2-ylamino)-2-oxo-N'phenylacetohydrazonoyl cyanide (12a) and 2-(3-(oxazol-2-ylcarbamoyl)-5,6-dihydro-4H-cyclopenta[b]thiophen-2-ylamino)-2-oxo- $\mathrm{N}^{\prime}$-phenylacetohydrazonoyl cyanide (12b). - To a cold solution $\left(0-5{ }^{\circ} \mathrm{C}\right)$ of either compound $4 \mathbf{a}(0.665 \mathrm{~g}, 0.002 \mathrm{~mol})$ or $4 \mathbf{b}(0.633 \mathrm{~g}, 0.002 \mathrm{~mol})$ in abs. ethanol $(50 \mathrm{~mL})$ containing sodium hydroxide $(0.08 \mathrm{~g}, 0.002 \mathrm{~mol})$, benzenediazonium chloride (11) $(0.002 \mathrm{~mol})$ [prepared by adding an aqueous sodium nitrite solution $(0.138 \mathrm{~g}, 0.002$ $\mathrm{mol})$ to a cold solution of aniline $(0.002 \mathrm{~mol})$ with an appropriate amount of concentrated $\mathrm{HCl}$, at $0-5{ }^{\circ} \mathrm{C}$, with continuous stirring] was added under continuous stirring. The reaction mixture was stirred at room temperature for an additional $4 \mathrm{~h}$ and the solid product so formed was collected by filtration and recrystallized from 1,4-dioxane.

2-(4-(Aminothiazol-2-yl)-6,7-dihydro-5H-cyclopenta[4,5]thieno[2,3-d]pyrimidin-2-yl) acetonitrile (13a) and 2-(4-(amino-oxazol-2-yl)-6,7-dihydro-5H-cyclopenta[4,5]thieno[2,3-d]pyrimidin2-yl)acetonitrile (13b). - A suspension of either $5 \mathbf{a}(0.663 \mathrm{~g}, 0.002 \mathrm{~mol})$ or $5 \mathbf{b}(0.631 \mathrm{~g}, 0.002$ mol) in sodium ethoxide $(50 \mathrm{~mL}, 0.002 \mathrm{~mol})$ was heated over a boiling water bath for $6 \mathrm{~h}$ and then left to cool. The solid product was formed upon pouring it into ice/water containing a few drops of concentrated hydrochloric acid (until $\mathrm{pH}=6$ ); it was collected by filtration and recrystallized from absolute ethanol.

2-(4,6-Diamino-5-cyanopyridin-2-ylamino)-N-(thiazol-2-yl)-5,6-dihydro-4H-cyclopenta[b] thiophene-3-carboxamide (15a), 2-(4,6-diamino-5-cyanopyridin-2-yl-amino) $\mathrm{N}$-(oxazol-2-yl)5,6dihydro-4H-cyclopenta[b] thiophene-3-carboxamide (15b), 2-(4-amino-5-cyano-6-hydroxylpyridin-2-ylamino)-N-(thiazol-2-yl)-5,6-dihydro-4H-cyclopenta[b]thiophene-3-carboxamide (15c) and 2-(4-amino-5-cyano-6-hydroxypyridine-2-ylamino)-N-(oxazol-2-yl)-5,6-dihydro-4Hcyclopenta[b]thiophene-3-carboxamide (15d). - To a suspension of either $5 \mathrm{a}(0.663 \mathrm{~g}, 0.002 \mathrm{~mol})$ or $5 \mathbf{b}(0.631 \mathrm{~g}, 0.002 \mathrm{~mol})$ in sodium ethoxide $(0.002 \mathrm{~mol}, 50 \mathrm{~mL})$, either ethyl cyanoacetate $(0.226 \mathrm{~g}, 0.002 \mathrm{~mol})$ or malononitrile $(0.132 \mathrm{~g}, 0.002 \mathrm{~mol})$ was added. The reaction mixture was heated under reflux for $3 \mathrm{~h}$. It was then poured onto an ice/water mixture containing a few drops of concentrated hydrochloric acid. The formed solid product was collected by filtration and recrystallized from absolute ethanol.

2-(Amino(2-oxo-2H-chromen-3-yl)methyleneamino)-N-(thiazol-2-yl)-5,6-dihydro-4Hcyclopenta[b]thiophene-3-carboxamide (16a) and 2-(amino(2-oxo-2H-chromen-3-yl)-methyleneamino)-N-(oxazol-2-yl)-5,6-dihydro-4H-cyclopenta[b]thiophene-3-carboxamide (16b). - To a solution of either compound $5 \mathbf{a}(0.663 \mathrm{~g}, 0.002 \mathrm{~mol})$ or $5 \mathbf{b}(0.631 \mathrm{~g}, 0.002 \mathrm{~mol})$ in 1,4-dioxane $(40 \mathrm{~mL})$ containing piperidine $(0.50 \mathrm{~mL})$, salicyladehyde $(0.244 \mathrm{~g}, 0.002 \mathrm{~mol})$ was added. The reaction mixture was heated under reflux for $4 \mathrm{~h}$ and then evaporated under vacuum. The 
remaining product was triturated with absolute ethanol and the crude solid product was collected by filtration and recrystallized from 1,4-dioxane.

4-(2-Amino-thiazol-2-yl)-2-(2-oxo-2H-chromene-3-yl)-6,7-dihydro-5H-cyclopenta[4,5] thieno[2,3-d]pyrimidine (17a) and 4-(2-aminooxazol-2-yl)-2-(2-oxo-2H-chromene-3-yl)-6,7-dihydro-5H-cyclopenta[4,5]thieno[2,3-d]pyrimidine (17b). - Method A. A solution of either compound 16a $(0.872 \mathrm{~g}, 0.002 \mathrm{~mol})$ or $16 \mathrm{~b}(0.84 \mathrm{~g}, 0.002 \mathrm{~mol})$ in dimethylformamide $(40 \mathrm{~mL})$ containing a catalytic amount of triethylamine $(0.5 \mathrm{~mL})$ was heated under reflux for $3 \mathrm{~h}$. It was then poured into an ice/water mixture containing a few drops of conc. $\mathrm{HCl}$. The formed solid product was collected by filtration and recrystallized from 1,4-dioxane.

Method B. To a solution of either compound $5 \mathrm{a}(0.663 \mathrm{~g}, 0.002 \mathrm{~mol})$ or $5 \mathbf{b}(0.631 \mathrm{~g}, 0.002$ $\mathrm{mol})$ in sodium ethoxide $(0.002 \mathrm{~mol}, 50 \mathrm{~mL})$, salicyladehyde $(0.244 \mathrm{~g}, 0.002 \mathrm{~mol})$ was added. The reaction mixture was heated under reflux for $4 \mathrm{~h}$ and then evaporated under vacuum. The remaining product was triturated with absolute ethanol and the solid product so formed was collected by filtration and recrystallized from 1,4-dioxane.

\section{Antitumor activity tests: materials and methods}

Fetal bovine serum (FBS) and $L$-glutamine were purchased from Gibco Invitrogen Co. (UK) while RPMI-1640 medium was from Cambrex (USA). Dimethyl sulfoxide (DMSO), doxorubicin, streptomycin, penicillin and sulforhodamine B (SRB) were all from Sigma Chemical Co. (USA).

Three different human tumor cell lines were used: MCF-7 (breast adenocarcinoma), NCI-H460 (non-small cell lung cancer) and SF-268 (CNS cancer). MCF-7 was obtained from the European Collection of Cell Cultures (ECACC, Salisbury, UK) while NCI-H460, SF-268 and normal fibroblast cells (WI-38) were kindly donated by the National Cancer Institute (NCI, Cairo, Egypt).

Cell lines grew as monolayers and were routinely maintained in RPMI-1640 medium supplemented with $5 \%$ heat-inactivated FBS, $2 \mathrm{mmol} \mathrm{L}^{-1}$ glutamine and antibiotics (penicillin $100 \mathrm{U} \mathrm{mL}^{-1}$ and streptomycin $100 \mathrm{mg} \mathrm{mL}^{-1}$ ), at $37^{\circ} \mathrm{C}$ in a humidified atmosphere containing $5 \% \mathrm{CO}_{2}$. Exponentially growing cells were obtained by plating $1.5 \times 10^{5}$ cell $\mathrm{mL}^{-1}$ for MCF-7 and SF-268 and $0.75 \times 10^{4}$ cell mL $\mathrm{mL}^{-1}$ for NCI-H460. This was followed by $24-\mathrm{h}$ incubation. The influence of DMSO as a solvent on the growth of cell lines was evaluated in all experiments. It was performed by exposing untreated control cells to the maximum concentration of DMSO used in each assay (0.5\%).

The in vitro tumor cell growth assay was performed according to the procedure described by the National Cancer Institute (USA) (13). This method uses the protein-binding dye sulforhodamine B to indicate cell growth. Afterwards, exponentially growing cells in 96-well plates were exposed to five serial dilutions of each compound, starting from a maximum concentration of $150 \mathrm{mmol} \mathrm{L}^{-1}$ for $48 \mathrm{~h}$. Following this exposure period, adherent cells were fixed, washed and stained. The bound stain was then dissolved in DMSO and absorbance was measured at $492 \mathrm{~nm}$ in a plate reader (Power wave XS, Bio-Tek Instruments, USA). For each test compound and cell line, a dose-dependent curve was established. GI $_{50}$ (concentration of the compound that inhibits $50 \%$ of net cell growth) was calculated (14). Doxorubicin was used as a reference drug (positive control). 


\section{RESULTS AND DISCUSSION}

\section{Chemistry}

Syntheses of the new heterocyclic compounds, thiophene, pyrimidine, coumarin, pyrazole and pyridine derivatives, are depicted in Schemes 1-4. Elucidation of their structures was based on analytical and spectral data.

Synthesis of compounds $\mathbf{2} \mathbf{a}, \mathbf{b}-\mathbf{4} \mathbf{a}, \mathbf{b}$ and $\mathbf{5 a} \mathbf{a}, \mathbf{b}$ is displayed in Scheme 1 . The reaction of ethyl cyanoacetate with either 2-aminothiazole (1a) or 2-aminoxazole (1) $\mathbf{b})$ yielded the acetamido derivative of each thiazole and oxazole ring $(\mathbf{2} \mathbf{a}, \mathbf{b})$, respectively. The latter, upon reaction with cyclopentanone in the presence of elemental sulfur and a catalytic amount of triethylamine, gave thiophene derivatives $\mathbf{3 a}, \mathbf{b}$, which were directed toward the reaction with either ethyl cyanoacetate or malononitrile, and yielded two pairs of carboxamide derivatives $\mathbf{4 a}, \mathbf{b}$ and $\mathbf{5 a}, \mathbf{b}$, respectively.

Reactivity of the first pair of carboxamide derivatives $4 \mathbf{a}, \mathbf{b}$ was studied through some different types of reactions. Thus, internal cyclization was introduced to give fused pyrimidine derivatives $\mathbf{6} \mathbf{a}, \mathbf{b}$. The structure of compounds $\mathbf{6} \mathbf{a}, \mathbf{b}$ was verified by elemental analysis and spectral data. In compound $6 \mathbf{a}$, the ${ }^{1} \mathrm{H}$ NMR spectrum indicated the presence of a multiplet at $\delta 1.96-2.08 \mathrm{ppm}$, which could be assigned to $3 \mathrm{CH}_{2}$ groups. A singlet at $\delta$ 3.08-3.12 ppm indicated the presence of the $\mathrm{CH}_{2}$ side group and a multiplet at $\delta 6.62-6.73$ ppm corresponded to $2 \mathrm{H}$ of the thiazole ring. The IR spectrum of compound $6 \mathrm{a}$ showed disappearance of $2 \mathrm{NH}$ stretching signals. Compounds $\mathbf{4 a}, \mathbf{b}$ also reacted with salicylaldehyde in the presence of piperidine to form coumarin derivatives $\mathbf{7 a}, \mathbf{b}$. For compound $\mathbf{7 a}$, the ${ }^{1} \mathrm{H}$ NMR spectrum showed a multiplet at $\delta 1.79-1.86 \mathrm{ppm}$ from the presence of $3 \mathrm{CH}_{2}$ groups, a multiplet at $\delta 6.65-6.75$ ppm corresponding to $2 \mathrm{H}$ of the thiazole ring, a singlet at $\delta 6.88 \mathrm{ppm}$ corresponding to $1 \mathrm{H}$ of the coumarin ring, a multiplet at $\delta 7.34-7.49 \mathrm{ppm}$ corresponding to $4 \mathrm{H}$ of the benzene ring and two singlets, $\mathrm{D}_{2} \mathrm{O}$-exchangeable at $\delta 8.39$ and $8.56 \mathrm{ppm}$ corresponding to $2 \mathrm{H}$ of $2 \mathrm{NH}$ groups. Furthermore, the reaction of compounds $\mathbf{4} \mathbf{a}, \mathbf{b}$ with either hydrazine hydrate or phenylhydrazine afforded compounds $8 \mathbf{a}-\mathbf{d}$. Pyrimidine derivatives $9 \mathbf{a}-\mathbf{d}$ were obtained through the reaction of compounds $4 \mathbf{a}, \mathbf{b}$ with either urea or thiourea in sodium ethoxide. ${ }^{1} \mathrm{H}$ NMR spectrum of compound 9a showed a multiplet at $\delta 1.83-1.94 \mathrm{ppm}$ that indicated $3 \mathrm{CH}_{2}$ groups, a singlet at $\delta 3.96 \mathrm{ppm}$ corresponding to $2 \mathrm{H}$ of the pyrimidine ring, a singlet, $\mathrm{D}_{2} \mathrm{O}$-exchangeable at $\delta 4.51 \mathrm{ppm}$ corresponding to $2 \mathrm{H}$ of the amino group, a multiplet at $\delta 6.61-6.68 \mathrm{ppm}$ corresponding to $2 \mathrm{H}$ of the thiazole ring and two singlets, $\mathrm{D}_{2} \mathrm{O}$-exchangeable at $\delta 8.53$ and 8.71 ppm corresponding to $2 \mathrm{H}$ of $2 \mathrm{NH}$ groups. In the reaction of compounds $4 \mathbf{a}, \mathbf{b}$ with either benzaldehyde or benzenediazonium chloride (11), compounds $\mathbf{1 0} \mathbf{a}, \mathbf{b}$ and $\mathbf{1 2} \mathbf{a}, \mathbf{b}$ were formed, respectively. ${ }^{1} \mathrm{H}$ NMR spectrum of compound 10a indicated a multiplet at $\delta 1.83-1.96 \mathrm{ppm}$ which represented $3 \mathrm{CH}_{2}$ groups, a singlet at $\delta 5.18 \mathrm{ppm}$ corresponding to $1 \mathrm{H}$ of $=\mathrm{CH}$ group, a multiplet at $\delta 6.33-6.58 \mathrm{ppm}$ corresponding to $2 \mathrm{H}$ of the thiazole ring, a multiplet at $\delta 7.35-7.48$ ppm corresponding to $5 \mathrm{H}$ of the benzene ring and two singlets, $\mathrm{D}_{2} \mathrm{O}$-exchangeable at $\delta$ 8.37 and $8.52 \mathrm{ppm}$ corresponding to $2 \mathrm{H}$ of $2 \mathrm{NH}$ groups.

Also, reactivity of the second pair of carboxamide derivatives $\mathbf{5} \mathbf{a}, \mathbf{b}$ was used. They underwent ready cyclization when heated in a sodium ethoxide solution, leading to the corresponding pyrimidine derivatives $\mathbf{1 3} \mathbf{a}, \mathbf{b}$. Thus, the ${ }^{1} \mathrm{H}$ NMR spectrum of compound 13a showed a multiplet at $\delta 1.76-1.85 \mathrm{ppm}$ from the $3 \mathrm{CH}_{2}$ groups, a singlet at $\delta 3.12-3.19$ 
ppm that indicated the presence of the $\mathrm{CH}_{2}$ side group, a multiplet at $\delta 6.51-6.63$ ppm corresponding to $2 \mathrm{H}$ of the thiazole ring and a singlet $\mathrm{D}_{2} \mathrm{O}$-exchangeable at $\delta 8.11$ ppm corresponding to $1 \mathrm{H}$ of the $\mathrm{NH}$ group. Moreover, ${ }^{13} \mathrm{C}$ NMR of the same compound showed signals at 21.2, 22.8, 25.4, 38.2 (cyclopentyl $\mathrm{C}, \mathrm{CH}_{2}$ side group), 121.4 (CN), 123.1, 125.4, 127.3, $130.4,132.9,138.6,143.3,146.4$ ppm (thiophene C, thiazole C, pyrimidine C). Furthermore, when compounds $\mathbf{5} \mathbf{a}, \mathbf{b}$ reacted with either ethyl cyanoacetate or malononitrile in sodium ethoxide, they formed pyridine derivatives 15a-d, through the formation of intermediate compounds 14a-d. ${ }^{1} \mathrm{H}$ NMR spectrum of 15a showed a multiplet at $\delta 1.81-1.93$ ppm assignable to $3 \mathrm{CH}_{2}$ groups, two singlets $\mathrm{D}_{2} \mathrm{O}$-exchangeable at $\delta 4.33$ and $4.52 \mathrm{ppm}$ that indicated the presence of $4 \mathrm{H}$ of $2 \mathrm{NH}_{2}$ groups, a multiplet at $\delta 6.61-6.78 \mathrm{ppm}$ corresponding to $2 \mathrm{H}$ of the thiazole ring and $1 \mathrm{H}$ of the pyridine ring and two singlets $\mathrm{D}_{2} \mathrm{O}$-exchangeable at $\delta$ 8.53 and $8.91 \mathrm{ppm}$ indicating the presence of $2 \mathrm{H}$ for $2 \mathrm{NH}$ groups. Finally, the reaction of $5 \mathbf{a}, \mathbf{b}$ with salicylaldehyde took two specific pathways. The first was carried out in piperidine affording compounds $\mathbf{1 6 a}, \mathbf{b}$. The other products underwent cyclization to form fused pyrimidine derivatives $\mathbf{1 7} \mathbf{a}, \mathbf{b}$ in dimethylformamide. The second pathway took place in a sodium ethoxide solution to form compounds $\mathbf{1 7} \mathbf{a}, \mathbf{b}$ directly. The analytical and spectral data of products $16 \mathbf{a}, \mathbf{b}$ and $\mathbf{1 7 a}, \mathbf{b}$ are consistent with the assigned structures.

\section{Effects on the growth of human tumor cell lines and QSAR}

Compounds $\mathbf{2} \mathbf{a}, \mathbf{b}-\mathbf{- 1 7} \mathbf{a}, \mathbf{b}$ were examined for their inhibitory effect on the in vitro growth of human tumor cell lines of different tumor types, namely, breast adenocarcinoma (MCF7), non-small cell lung cancer (NCI-H460) and CNS cancer (SF-268). All tested compounds inhibited the growth of the tested tumor cell lines in a dose-dependent manner (data not shown). The results from Table III show that fused pyrimidine acetonitrile derivatives $\mathbf{6} \mathbf{a}, \mathbf{b}$ exerted the highest inhibitory effect against all three tumor cell lines, comparable with the reference standard. Among the fused pyrimidine derivatives containing coumarin moiety, compounds $\mathbf{1 7} \mathbf{a}, \mathbf{b}$ showed the highest inhibitory effects against all three tumor cell lines. Compounds $\mathbf{2} \mathbf{a}, \mathbf{b}, \mathbf{1 3} \mathbf{a}, \mathbf{b}$ and $\mathbf{1 5} \mathbf{a}, \mathbf{b}, \mathbf{c}, \mathbf{d}$ showed moderate inhibitory effects against all three cancer cell lines, while the rest of the compounds (3a,b, 4a,b, 5a,b, 7a,b, 8a-d, 9a-d, 10a,b, $12 a, b$ and $16 a, b)$ showed low effects.

When comparing fused pyrimidine acetonitrile derivatives, it was found that compound $\mathbf{6 b}$ was slightly more effective than $\mathbf{6 a}$, possibly due to the presence of the oxazole ring in $\mathbf{6 b}$ instead of the thiazole ring in $\mathbf{6 a}$. On the other hand, for the fused pyrimidine derivatives containing coumarin moiety, it was found that compound $\mathbf{1 7 a}$ with the thiazole ring showed stronger effect than compound $\mathbf{1 7 b}$ containing the oxazole ring. Cyanoacetamide derivatives $\mathbf{2} \mathbf{a}$ and $\mathbf{2} \mathbf{b}$ showed comparable effects in the presence of the thiazole ring in compound $\mathbf{2} \mathbf{a}$ instead of oxazole ring in compound $\mathbf{2} \mathbf{b}$. The same was seen when comparing fused pyrimidine derivatives, 13a with the thiazole ring and $\mathbf{1 3 b}$ with the oxazole ring. Among pyridine derivatives $15 a-d$, compound $15 a$ was most effective against all three cell lines, possibly due to the presence of the $\mathrm{NH}_{2}$ group instead of $\mathrm{OH}$ group (in $15 \mathrm{c}$ and $15 \mathrm{~d}$ ), in addition to the presence of the thiazole ring instead of the oxazole ring (in 15b).

When comparing the amino-thiophene derivatives $\mathbf{3} \mathbf{a}, \mathbf{b}$, acetamido-thiophene derivatives $\mathbf{4} \mathbf{a}, \mathbf{b}$ and acetimido-thiophene derivatives $\mathbf{5} \mathbf{a}, \mathbf{b}$, it was found that these compounds showed almost the same effects despite structural differences (namely, the presence of $\mathrm{NH}_{2}$ in $3 \mathbf{a}, \mathbf{b}, \mathrm{CNCH}_{2} \mathrm{CONH}$ in $4 \mathbf{a}, \mathbf{b}$ and $\mathrm{CNCH}_{2} \mathrm{C}=\mathrm{N}\left(\mathrm{NH}_{2}\right)$ in $\left.\mathbf{5} \mathbf{a}, \mathbf{b}\right)$. On the other hand, 
M. Albratty et al:: Synthesis and antitumor activity of some novel thiophene, pyrimidine, coumarin, pyrazole and pyridine derivatives, Acta Pharm. 67 (2017) 15-33.

Table III. Effect of compounds $\mathbf{2} \boldsymbol{a}, \boldsymbol{b} \mathbf{- 1 7} \boldsymbol{a}, \boldsymbol{b}$ on the growth of three human tumor cell lines

\begin{tabular}{|c|c|c|c|c|}
\hline \multirow{2}{*}{ Compd. } & \multicolumn{4}{|c|}{$G I_{50}\left(\mathrm{mmol} \mathrm{L}^{-1}\right)^{\mathrm{a}}$} \\
\hline & MCF-7 & NCI-H460 & SF-268 & WI-38 \\
\hline $2 a$ & $23.7 \pm 7.4$ & $24.1 \pm 5.3$ & $28.5 \pm 5.9$ & NA \\
\hline $2 b$ & $27.1 \pm 8.9$ & $25.7 \pm 6.8$ & $26.2 \pm 4.9$ & NA \\
\hline $3 a$ & $44.6 \pm 7.8$ & $45.1 \pm 7.3$ & $44.3 \pm 7.7$ & $65.5 \pm 11.7$ \\
\hline $3 b$ & $41.9 \pm 7.9$ & $48.7 \pm 6.3$ & $42 \pm 8.3$ & NA \\
\hline $4 a$ & $51.3 \pm 11.8$ & $50.4 \pm 10.1$ & $49.5 \pm 11.5$ & $>100$ \\
\hline $4 b$ & $44.2 \pm 9.3$ & $46.1 \pm 9.3$ & $47.5 \pm 9.5$ & $>100$ \\
\hline $5 a$ & $48.0 \pm 9.9$ & $48.2 \pm 10.7$ & $44.7 \pm 9.5$ & $>100$ \\
\hline $5 b$ & $41.8 \pm 7.6$ & $43.5 \pm 6.5$ & $43.5 \pm 8.1$ & $38.1 \pm 12.5$ \\
\hline $6 a$ & $0.1 \pm 0.08$ & $0.25 \pm 0.4$ & $1.6 \pm 0.3$ & $15.1 \pm 8.3$ \\
\hline $6 b$ & $0.09 \pm 0.06$ & $0.07 \pm 0.03$ & $1.3 \pm 0.2$ & $14.4 \pm 7.6$ \\
\hline $7 a$ & $37.9 \pm 9.5$ & $36.4 \pm 8.7$ & $32.1 \pm 6.8$ & $22.7 \pm 11.3$ \\
\hline $7 \mathrm{~b}$ & $39.3 \pm 10.2$ & $34.6 \pm 8.8$ & $37.9 \pm 9.7$ & $49.1 \pm 14.4$ \\
\hline $8 a$ & $40.3 \pm 5.4$ & $41.1 \pm 7.6$ & $36.6 \pm 8.6$ & $28.3 \pm 12.3$ \\
\hline $8 b$ & $33.1 \pm 7.2$ & $34.5 \pm 5.9$ & $35.3 \pm 6.2$ & $21.1 \pm 13.5$ \\
\hline $8 c$ & $29.0 \pm 6.9$ & $30.0 \pm 7.4$ & $27.5 \pm 10.8$ & $22.6 \pm 11.5$ \\
\hline $8 d$ & $35.9 \pm 11.3$ & $39.2 \pm 122$ & $36.0 \pm 14.1$ & $23.1 \pm 14.8$ \\
\hline $9 a$ & $44.2 \pm 7.3$ & $46.6 \pm 6.4$ & $34.9 \pm 19.1$ & $12.7 \pm 11.8$ \\
\hline $9 b$ & $34.9 \pm 11.2$ & $41.0 \pm 8.8$ & $44.5 \pm 11.6$ & $29.3 \pm 10.1$ \\
\hline $9 \mathrm{c}$ & $36.7 \pm 6.9$ & $40.9 \pm 6.6$ & $42.4 \pm 9.3$ & NA \\
\hline $9 d$ & $41.2 \pm 8.6$ & $34.0 \pm 6.9$ & $39.7 \pm 8.7$ & NA \\
\hline $10 a$ & $42.7 \pm 13.9$ & $39.9 \pm 14.3$ & $32.3 \pm 5.3$ & NA \\
\hline $10 \mathrm{~b}$ & $39.2 \pm 6.8$ & $37.3 \pm 6.4$ & $35.9 \pm 6.9$ & NA \\
\hline $12 a$ & $45.2 \pm 12.8$ & $47.1 \pm 16.4$ & $38.5 \pm 21.1$ & NA \\
\hline $12 b$ & $38.4 \pm 7.7$ & $35.2 \pm 8.3$ & $37.6 \pm 8.9$ & NA \\
\hline $13 a$ & $21.7 \pm 6.9$ & $17.9 \pm 4.7$ & $21.5 \pm 5.3$ & $>100$ \\
\hline $13 b$ & $21.2 \pm 5.6$ & $18.0 \pm 3.9$ & $19.7 \pm 4.9$ & $>100$ \\
\hline $15 a$ & $16.2 \pm 6.9$ & $17.3 \pm 7.8$ & $15.1 \pm 9.9$ & $>100$ \\
\hline $15 b$ & $25.2 \pm 6.8$ & $25.3 \pm 9.4$ & $26.9 \pm 11.9$ & $>100$ \\
\hline $15 c$ & $19.9 \pm 12.8$ & $23.1 \pm 11.4$ & $30.2 \pm 15.3$ & $>100$ \\
\hline $15 d$ & $20.2 \pm 6.3$ & $27.1 \pm 8.3$ & $25.5 \pm 9.5$ & $>100$ \\
\hline $16 a$ & $41.1 \pm 14.8$ & $43.3 \pm 16.4$ & $39.8 \pm 6.9$ & NA \\
\hline $16 b$ & $39.7 \pm 8.5$ & $37.4 \pm 9.9$ & $32.6 \pm 10.5$ & NA \\
\hline $17 a$ & $3.1 \pm 0.09$ & $2.3 \pm 1.4$ & $2.9 \pm 1.1$ & $>100$ \\
\hline $17 \mathrm{~b}$ & $6.2 \pm 1.4$ & $3.4 \pm 0.7$ & $4.8 \pm 0.9$ & $>100$ \\
\hline Doxorubicin & $0.04 \pm 0.008$ & $0.09 \pm 0.008$ & $0.09 \pm 0.007$ & $>100$ \\
\hline
\end{tabular}

NA - not applicable

Results are given in concentrations that were able to cause $50 \%$ of cell growth inhibition $\left(G_{50}\right)$ after a continuous exposure for $48 \mathrm{~h}$.

${ }^{\text {a }}$ Mean \pm SEM of three-independent experiments performed in duplicate. 
when comparing coumarin derivatives $\mathbf{7 a}, \mathbf{b}$, pyrazole derivatives $\mathbf{8} \mathbf{a}-\mathbf{d}$ and pyrimidine derivatives $9 a-d$, it was found that compound $\mathbf{8 c}$ was highly effective. This may be due to the presence of the pyrazole ring instead of either coumarin ring in compounds $\mathbf{7 a}, \mathbf{b}$ or pyrimidine ring in compounds $\mathbf{9 a}$-d, in addition to the phenyl group instead of $\mathrm{H}$ (in $\mathbf{8} \mathbf{a}, \mathbf{b}$ ) and thiazole ring instead of oxazole ring (in 8d).

Finally, when comparing benzylidine derivatives $\mathbf{1 0 a}, \mathbf{b}$, phenylhydrazone derivatives $\mathbf{1 2} \mathbf{a}, \mathbf{b}$ and coumarin derivatives $\mathbf{1 6} \mathbf{a}, \mathbf{b}$, it was found that these compounds may be of almost the same efficacy despite the presence of the benzylidine side chain in compounds $\mathbf{1 0 a}, \mathbf{b}$, phenylhydrazo side chain in compounds $\mathbf{1 2} \mathbf{a}, \mathbf{b}$ and coumarin moiety in compounds $\mathbf{1 6} \mathbf{a}, \mathbf{b}$.

\section{CONCLUSIONS}

Among the newly synthesized products, fused pyrimidine acetonitrile derivatives $6 \mathrm{a}$ and $\mathbf{6 b}$ showed high inhibitory activity against all the three tumor cell lines, MCF-7, NCIH460 and SF-268, comparable to that of doxorubicine They were followed by fused pyrimidine derivatives containing the coumarin moiety, $\mathbf{1 7} \mathbf{a}$ and $\mathbf{1 7} \mathbf{b}$.

Acknowledgments. - The authors thank the research group working at the Medicinal Department at the National Research Center, Dokki, Egypt, for recording the pharmacological data of the synthesized products as well as the Poison Control and Medical Forensic Chemistry Center Team, Jazan Health, Jazan City, Kingdom of Saudi Arabia, for recording the analytical and spectral data of the newly synthesized compounds.

\section{REFERENCES}

1. R. Romagnoli, P. G. Baraldi, C. Lopez-Cara, M. K. Salvador, D. Preti, M. A. Tabrizi, J. Balzarini, P. Nussbaumer, M. Bassetto, A. Brancale, X. H. Fu, Y. Gao, J. Li, S. Z. Zhang, E. Hamel, R. Bortolozzi, G. Basso and G. Viola, Design, synthesis and biological evaluation of 3,5-disubstituted 2- aminothiophene derivatives as a novel class of antitumor agents, Bioorg. Med. Chem. 22 (2014) 5097-5109; DOI: 10.1016/j.bmc. 2013.12.030.

2. R. Romagnoli, P. G. Baraldi, M. D. Carrion, C. L. Cara, D. Perti, F. Fruttarolo, M. G. Pavani, M. A. Tabrizi, M. Tolomio, S. Grimaudo, A. Di Cristina, J. Balzarini, J. A. Hadfield, A. Bracale and E. Hamel, Synthesis and biological evaluation of 2- and 3-aminobenzo[b]thiophene derivatives as antimitotic agents and inhibitors of tubulin polymerization, J. Med. Chem. 50 (2007) 2273-2277; DOI: $10.1021 / j m 070050 f$.

3. C. E. Stephens, T. M. Felder, J. W. Sowell, G. Andrei, J. Balzarini, R. Snoeck and E. De Clercq, Synthesis and antiviral/antitumor evaluation of 2-amino- 2-carboxamido-3-aryl-sulfonylthiophenes and related compounds as a new class of diarylsulfones, Bioorg. Med. Chem. 9 (2001) 1123-1132; DOI: $10.1016 /$ S0968-0896(00)00333-3.

4. S. Sulzer-Mosse, F. Cederbaum, C. Lamberth, G. Berthon, J. Umarye, V. Grasso, A. Schlereth, M. Blum and R. Waldmeier, Synthesis and fungicidal activity of N-thiazol-4-yl-salicylamides, a new family of anti-oomycete compounds, Bioorg. Med. Chem. 23 (2015) 2129-2138; DOI: 10.1016/j. bmc.2015.03.007.

5. V. Padmavathi, C. Prema Kumari, B. C. Venkatesh and A. Padmaja, Synthesis and antimicrobial activity of amido linked pyrrolyl and pyrazolyl-oxazoles, thiazoles and imidazoles, Eur. J. Med. Chem. 46 (2011) 5317-5326; DOI: 10.1016/j.ejmech.2011.08. 032. 
6. M. B. Dewal, A. S. Wani, C. Vidaillac, D. Oupický, M. J. Rybak and S. M. Firestine, Thieno[2,3-d] pyrimidinedione derivatives as antibacterial agents, Eur. J. Med. Chem. 51 (2012) 145-153; DOI: 10.1016/j.ejmech.2012.02.035.

7. A. T. Mavrova, D. Wesselinova, J. A. Tsenov and L. A. Lubenov, Synthesis and antiproliferative activity of some new thieno[2,3-d]pyrimidine-4-(3H)-ones containing 1,2,4-triazole and 1,3,4-thiadiazole moiety, Eur. J. Med. Chem. 86 (2014) 676-683; DOI: 10.1016/j.ejmech.2014.09.032.

8. F. Chimenti, B. Bizzarri, A. Bolasco, D. Secci, P. Chimenti, S. Carradori, A. Granese, D. Rivanera, D. Lilli, A. Zicari, M. M. Scaltrito and F. Sisto, A novel class of selective anti-Helicobacter pylori agents 2-oxo-2H-chromene-3-carboxamide derivatives, Bioorg. Med. Chem. Lett. 17 (2007) 30653071; DOI: 10.1016/j.bmcl. 2007. 03.050.

9. S. Bindi, D. Fancelli, C. Alli, D. Berta, J. A. Bertrand, A. D. Cameron, P. Cappella, P. Carpinelli, G. Cervi, V. Croci, M. D’Anello, B. Forte, M. L. Giorgini, A. Marsiglio, J. Moll, E. Pesenti, V. Pittalà, M. Pulici, F. Riccardi-Sirtori, F. Roletto, C. Soncini, P. Storici, M. Varasi, D. Volpi, P. Zugnoni and P. Vianello, Thieno[3,2-c]pyrazoles: a novel class of Aurora inhibitors with favorable antitumor activity, Bioorg. Med. Chem. 18 (2010) 7113-7120; DOI: 10.1016/j.bmc.2010.07.048.

10. S. Ulloora, R. Shabaraya, R. Ranganathan and A. V. Adhikari, Synthesis, anticonvulsant and antiinflammatory studies of new 1,4-dihydropyridin-4-yl-phenoxyacetohydrazones, Eur. J. Med. Chem. 70 (2013) 341-349; DOI: 10.1016/j. ejmech.2013.10. 010.

11. A. A. Napoleon, F. R. N. Khan, E. D. Jeong and E. H. Chung, Potential antitubercular agents: Hexahydro-3-phenyl indazol-2-yl-(pyridine-4-yl) methanones from antitubercular drug isoniazid and bis(substituted benzylidene) cycloalkanones, Chin. Chem. Lett. 26 (2015) 567-571; DOI: 10.1016/j.cclet.2015.01.008.

12. S. Malik, P. Ahuja, K. Sahu and S. A. Khan, Design and synthesis of new 3-(benzo [d] isoxazol3yl)-1-substituted pyrrolidine-2,5-dione derivatives as anti-convulsants, Eur. J. Med. Chem. 84 (2014) 42-50; DOI: 10.1016/j.ejmech.2014.07.016

13. P. Skehan, R. Storeng, D. Scudiero, A. Monks, J. McMahon, D. Vistica, J. T. Warren, H. Bokesch, S. Kenney and M. R. Boyd, New colorimetric cytotoxicity assay for anticancer-drug screening, J. Natl. Cancer Inst. 82 (1990) 1107-1112; DOI: 10.1093/jnci/82.13.1107.

14. A. Monks, D. Scudiero, P. Skehan, R. Shoemaker, K. Paull, D. Vistica, C. Hose, J. Langley, P. Cronise, A. Vaigro-Wolff, M. Gray-Goodrich, H. Campbell, J. Mayo and M. J. Boyd, Feasibility of a high-flux anticancer drug screen using a diverse panel of cultured human tumor cell lines, J. Natl. Cancer Inst. 83 (1991) 757-766; DOI: 10.1093/jnci/83.11.757. 